\title{
Carboxypeptidase $Y$ assisted disulfide-bond identification with linearized database search
}

\author{
Jiali Qiang ${ }^{1,2}$, Zhimin $\mathrm{Xu}^{1,2}$, Yunxia $\mathrm{Li}^{1}$, Hongbin Wang ${ }^{3 *}$, Yaoyang Zhang ${ }^{1 *}$
}

${ }^{1}$ Interdisciplinary Research Center on Biology and Chemistry, Shanghai Institute of Organic Chemistry, Chinese Academy of Sciences, 100 Haike Rd., Pudong, Shanghai 201210, China

${ }^{2}$ University of Chinese Academy of Sciences, Beijing 100049, China

${ }^{3}$ The College of Biotechnology, Tianjin University of Science and Technology, Tianjin 300457, China

* Corresponding author:

Dr. Hongbin Wang

Phone: +86-22-60601958

E-mail: whb@tust.edu.cn

Dr. Yaoyang Zhang

Phone: +86-21-68582550

Fax: +86-21-64166128

E-mail:zyy@sioc.ac.cn 


\section{Supporting Information:}

The following supporting information is available free of charge at ACS website http://pubs.acs.org

\section{Table of Contents}

Experimental Details

CADI sample preparation for standard proteins

CADI sample preparation for HeLa cells

Reducible cysteine analysis

LC-MS/MS analysis

Linearized database for Mascot

Linearized database for MaxQuant

FDR control with Mascot

Database search with MaxQuant

Supplementary Results

Linearization of disulfide-linked peptides

Decoy database for CADI

Reference

Supplementary figures

Figure S1 Linearization of disulfide-bonded peptides and their fragmentation in tandem $M S$.

Figure S2 Identification of disulfide bonds using different methods. 
Figure S3 Database used for CADI.

Figure S4 CADI removes linear peptides of HEK293T digest.

Figure S5 Comparison of peptides with different sensitivity to CPY.

Figure S6 Tandem mass spectra of disulfide bonds from Rituximab.

Figure S7 Validation of disulfide bonds identified from RNase A using synthetic peptides.

Supplementary table

Supplementary Table S1. Sequences of synthetic peptides. 


\section{Experimental Details}

\section{CADI sample preparation for standard proteins}

Rituximab alone or a 4-protein-mixture (1:1:1:1,w/w/w/w) (ovalbumin, lysozyme, transferrin and RNase A) was dissolved in $8 \mathrm{M}$ urea, $100 \mathrm{mM}$ Tris, $\mathrm{pH}$ 6.5, in the presence of $2 \mathrm{mM}$ NEM at $37{ }^{\circ} \mathrm{C}$ for $2 \mathrm{~h}$. The total protein concentrations were controlled at $2 \mu \mathrm{g} / \mu \mathrm{L}$. For Rituximab, protein was first digested with trypsin $(1: 100, \mathrm{w} / \mathrm{w})$ for $16 \mathrm{~h}$ at $37^{\circ} \mathrm{C}$, followed by chymotrypsin $(1: 100, \mathrm{w} / \mathrm{w})$ for $12 \mathrm{~h}$ at $25{ }^{\circ} \mathrm{C}$. In addition, Rituximab was also digested with trypsin or chymotrypsin separately. The digestion was quenched by adding $5 \%$ FA. The 4-proteinmixture was divided into three equal aliquots. Each aliquot was treated with trypsin $(1: 100$, $\mathrm{w} / \mathrm{w})$ at $37{ }^{\circ} \mathrm{C}$ for $16 \mathrm{~h}$; or Lys-N $(1: 100, \mathrm{w} / \mathrm{w})$ at $37{ }^{\circ} \mathrm{C}$ for $4 \mathrm{~h}$ followed by trypsin $(1: 100$, $\mathrm{w} / \mathrm{w})$ at $37{ }^{\circ} \mathrm{C}$ for $16 \mathrm{~h}$; or consequential treatment of Lys-N $(1: 100, \mathrm{w} / \mathrm{w})$ at $37^{\circ} \mathrm{C}$ for $4 \mathrm{~h}$ followed by trypsin $(1: 100, \mathrm{w} / \mathrm{w})$ at $37^{\circ} \mathrm{C}$ for $16 \mathrm{~h}$ and Glu-C $(1: 100,10 \mathrm{~h})$ at $25^{\circ} \mathrm{C}$ for $10 \mathrm{~h}$, respectively. The resulting peptides were centrifuged at $15,000 \mathrm{~g}$ for $10 \mathrm{~min}$. The supernatants were desalted with $\mathrm{C}_{18}$ StageTips, and dried in a vacuum concentrator. Then the peptides were either analyzed directly by shotgun and pLink, or treated with carboxypeptidase Y under the same condition as described above.

\section{CADI sample preparation for HeLa cells}

HeLa cell lysate was digested using trypsin and Glu-C sequentially as described above. The desalted digests were redissolved in $10 \%$ formic acid and then loaded onto a SCX column (4.6 $\mathrm{mm}$ inner diameter, $250 \mathrm{~mm}$ length, $5 \mu \mathrm{m}$ resin). SCX solvent A consisted of $0.05 \%$ formic 
acid in $20 \%$ acetonitrile, and solvent $\mathrm{B}$ consisted of $0.05 \%$ formic acid, $0.5 \mathrm{M} \mathrm{NaCl}$ in $20 \%$ acetonitrile. The SCX gradient ran as below: 0-8 $\min (2-8 \% \mathrm{~B}) ; 8-14 \min (8-20 \% \mathrm{~B}) ; 14-28$ $\min (20-40 \%$ B); $28-38 \min (40-60 \%$ B); 38-48 $\min (60-90 \%$ B); 48-56 min $(90 \%$ B); and 56-60 $\min (90-0 \%$ B). Fractions were collected every two minutes, and a total of 30 fractions were collected. The last ten SCX fractions which predominantly contained large and highly charged peptides $(\mathrm{z}>3)^{1}$ were desalted with $\mathrm{C}_{18}$ StageTips, and dried in a vacuum concentrator. Peptides were further divided into two aliquots. The first aliquot was directly analyzed by shotgun analysis, and the data were processed using pLink. The remaining peptides were treated with carboxypeptidase $\mathrm{Y}$, and subjected to CADI under the same condition as described above.

\section{Reducible cysteine analysis}

HeLa cell lysate was dissolved in $8 \mathrm{M}$ urea and $2 \mathrm{mM}$ NEM (100 mM Tris $\mathrm{pH}$ 6.5) and incubate at $37{ }^{\circ} \mathrm{C}$ for $2 \mathrm{~h}$. The buffer was exchanged by $8 \mathrm{M}$ urea for 2 times. $5 \mathrm{mM}$ TCEP was added for $30 \mathrm{~min}$ at $25^{\circ} \mathrm{C}$. The reduced cysteine was further alkylated with $10 \mathrm{mM}$ IAA. The proteins were subjected to a trypsin digestion and shotgun analysis. The IAA modified Cys was defined as reducible Cys, which included those derived from disulfide bonds. These reducible Cys residues were used to construct a sequence database for HeLa cell CADI analysis.

\section{LC-MS/MS analysis}

Samples were analyzed on an online LC-MS system consisting of an Easy nano-LC 1000 and a Q Exactive HF mass spectrometer (Thermo Scientific, Bremen, Germany). The peptides were separated on an in-house packed $\mathrm{C}_{18}$ capillary column $(15 \mathrm{~cm} ; 100 \mu \mathrm{m}$ inner diameter; $1.9 \mu \mathrm{m}$ 
resin (Dr. Maisch, Germany)) over a 60-min gradient. The MS data were acquired in a datadependent mode. For MS1, the scan range was 350 to $1,500 \mathrm{~m} / \mathrm{z}$ at a resolution of 60,000 , and the AGC target was $3 \times 10^{6}$. Precursor ions with $2-8$ charges were selected for tandem MS analysis. The MS2 configurations were different for peptide and protein samples. For standard peptide samples, the scan range was 200 to $2,000 \mathrm{~m} / \mathrm{z}$ at a resolution of 15,000 , the AGC target was $2 \times 10^{5}$, and the maximum injection time was $150 \mathrm{~ms}$. The dynamic exclusion time was 15 s. The protein samples used a scan range of 200 to $2,000 \mathrm{~m} / \mathrm{z}$ at a resolution of 30,000 . The AGC target was $1 \times 10^{5}$, and the maximum injection time was $45 \mathrm{~ms}$. The dynamic exclusion time was $30 \mathrm{~s}$. Both methods set a normalized collision energy of 27 for HCD.

\section{Linearized database for Mascot}

First, the protein sequences of interest were digested with proteases (e.g. trypsin) in silico. Other configurations, such as the maximum allowed number of missed cleavages, could be applied if necessary. Assuming that any two cysteine residues on the same protein could be connected, all possible disulfide-linked peptide pairs were generated. In the current study, peptides with only one disulfide bond were taken into consideration.

Then the sequences of each disulfide-linked peptides were linearized. Taken disulfidelinked peptides $\alpha$ and $\beta$ for example, peptide $\alpha$ keeps unchanged in the transformation, while peptide $\beta$ will become the carboxy-section of the pseudo-linear peptide. The first $\mathrm{N}$-terminal amino acid on $\beta$ peptide will act as the last pseudo $\mathrm{C}$-terminal amino acid. Other amino acids on $\beta$ peptide will be transformed following a same order. To distinguish free cysteine and disulfide-forming cysteine, an amino acid of pyrrolysine (Pyl, O), which doesn't exist in human 
proteome, was hijacked to represent disulfide-forming cysteine $\left(\mathrm{C}_{3} \mathrm{H}_{6} \mathrm{NO}_{2} \mathrm{~S}\right.$, monoisotopic mass: $120.0119 \mathrm{Da})$ in the Mascot configuration. During this linearization, all possible sequences with a varied number of $\mathrm{C}$-end amino acids (downstream of disulfide-forming cysteine) were linearized. When considering 0-2 C-end amino acid, a pair of Cys-containing peptides have 9 different linearized sequences. Notably, a further consideration of more than two C-end amino acid is totally compatible with CADI, however, such an addition will significantly increase database size and corresponding search time.

All possible sequences of a given pair of disulfide-linked peptides were assembled into a protein entry in the linearized protein database. Different sequences with a different number of C-end amino acids were separated by another hypothetical separator amino acid J. A customized enzyme CPY was set in Mascot to cut at the both ends of $\mathrm{J}$, thus generated all possible sequences for linearized peptide database search.

The decoy databases consisting of reverse-reverse (RR), forward-reverse (FR), and reverse-forward (RF), were generated in a similar manner. The reverse sequences were obtained by reversing the entire linearized sequences.

All databases were generated by using an in-house script written in $\mathrm{R}$ language.

\section{Linearized database for MaxQuant}

A fixed modification of $-117.1358 \mathrm{Da}$ on the hijacked $\mathrm{O}$ was added to mimic disulfideforming cysteine. We used $\mathrm{U}$, originally denoted for selenocysteine (Sec, $\mathrm{U}$ ), as a separator in our database. Sec presents at an extremely low frequency in the whole proteome (e.g. only 25 
sec-containing proteins in human ${ }^{2}$ ), thus has no impact on database search. A customized enzyme CPY was set in MaxQuant to cut at the both ends of U.

\section{FDR control with Mascot}

For the matches obtained in the hybrid decoy database (FR and RF), if the ratio between matched ions and amino acid number on the reverse chain was less than 0.32 or the length of reverse chain is shorter than 3 amino acids, FR and RF matches were reevaluated as FF. The false discovery rate (FDR) was controlled as previously described for cross-linking peptides ${ }^{3}$, ${ }^{4}$, using $F D R=\left(N_{U}-N_{F}\right) / N_{T}$. $\left(N_{T}\right.$ is the number of the spectra that match to peptide pairs in $\mathrm{FF}, N_{U}$ is the number of the spectra that match to peptide pairs in FR and RF, $N_{F}$ is the number of the spectra that match to peptide pairs in RR). The resulting disulfide bonds were filtered by controlling an FDR better than 0.05 .

\section{Database search with MaxQuant}

Raw CADI data were searched against a linearized database with MaxQuant software (version 1.6.0.1). A customized enzyme of CPY was set to cut at both the C-term and N-term of U without missed cleavage sites. The modification set-up was the same as in the Mascot. The peptide mass tolerances were set to 20 and $4.5 \mathrm{ppm}$ for the first and main searches, respectively. The MS/MS mass tolerance was set to $0.5 \mathrm{Da}$ for ITMS, and $20 \mathrm{ppm}$ for FTMS. The "requantify" and "match-between-run" options were selected. The false discovery rate (FDR) in MaxQuant was not enabled. The post-search quality control for MaxQuant was the same as for Mascot. 


\section{Supplementary results}

\section{Linearization of disulfide-linked peptides}

The linearization process was performed on disulfide-linked peptides $\alpha$ and $\beta$. The $\alpha$ and $\beta$ peptides were named based on the order of their appearances in a sequence database. Peptide $\alpha$ keeps unchanged in the transformation, while peptide $\beta$ will become the carboxy-section of the pseudo-linear peptide. The first N-terminal amino acid on $\beta$ peptide will act as the last pseudo-C-terminal amino acid. Other amino acids on $\beta$ peptide will be transformed following a same order (Fig. S1a). A hypothetical amino acid "O" (originally stands for a non-human amino acid of pyrrolysine) with a chemical formular of $\mathrm{C}_{3} \mathrm{H}_{6} \mathrm{NO}_{2} \mathrm{~S}$ was introduced to represent the disulfide-forming cysteine. A molecule of water was further removed at the pseudo-Cterminus to keep the chemical formula identical after linearization. For fragment ions in MS2 used for database search, all b-ions derived from peptides $\alpha$ keep unaffected after linearization, and $y$-ions only change their ion indexing numbers accordingly. For $\beta$ peptide, the actual b- or y-ions will be treated as pseudo-y- or pseudo-b-ions in the linearized peptide, respectively. Additionally, all ion indexing numbers on $\beta$ peptide need to be reassigned. After this transformation, all original fragment ions will match to a theoretical fragment value in the pseudo-linearized sequence (Fig. S1b - d).

\section{Decoy database for CADI}

As demonstrated by previous studies, the cross-linking peptide identification could be qualitycontrolled by using a decoy database $\mathrm{e}^{3,5}$. We implemented a similar model in our study, in which the whole searching database was consisting of four subgroups of sequences (Fig. S3a). The target database contains two froward (denoted by F) sequences for each pair of disulfide-linked 
peptides. The two types of hybrid decoy sequences have one forward sequence with the other reverse (denoted by R) sequence. The fourth decoy has two reverse sequences. Notably, the size of the decoy database (FR, RF, and RR) is three times as big as that of a target database (FF).

To evaluate if this database was useful, we first searched the CADI data of P10X against an E.coli protein database (243,748 total sequences) consisting of all four types of subdatabases. As there was no E.coli proteins truly presented in the sample, the random matches to the four sub-databases were quite comparable (Fig. S3b - d). After adding the standard peptide sequences to the above E.coli database, the identification of disulfide-linked peptides was significantly increased from the right database. In contrast, all decoy databases only returned much fewer random matches (Fig. 1c).

We further searched the CADI data of P10X against FF, RF, FR, or RR database separately (Fig. S3e). Interestingly, the independent search against the two hybrid databases (FR and RF) resulted in lots of identifications that could also be obtained from FF database search. This was because the fragment ions were still able to be matched to the F sequence in FR or RF database search. We next examined the details for those peptides that could be identified from more than one sub-databases (Fig. S3f). Our data showed that the F sequences identified in FF, or FR/RF databases normally contained a larger number of amino acids and fragment ions (Fig. S3g and h), while the R sequences were normally short. This meant though $\mathrm{R}$ could be identified in FR or FR databases, the $\mathrm{R}$ sequences contributed very little to the final identification. In summary, the target-decoy database strategy used in this study can help identify the true disulfide-linked peptides. 


\section{Reference}

1. Liu, F.; Rijkers, D. T.; Post, H.; Heck, A. J., Proteome-wide profiling of protein assemblies by cross-linking mass spectrometry. Nature methods 2015, 12 (12), 1179-84.

2. Guo, L.; Yang, W.; Huang, Q.; Qiang, J.; Hart, J. R.; Wang, W.; Hu, J.; Zhu, J.; Liu, N.; Zhang, Y., Selenocysteine-Specific Mass Spectrometry Reveals TissueDistinct Selenoproteomes and Candidate Selenoproteins. Cell Chem Biol 2018, 25 (11), $1380-1388$ e 4.

3. Yang, B.; Wu, Y. J.; Zhu, M.; Fan, S. B.; Lin, J.; Zhang, K.; Li, S.; Chi, H.; Li, Y. X.; $\quad$ Chen, H. F.; $\quad$ Luo, S. K.; $\quad$ Ding, Y. H.; $\quad$ Wang, L. H.; Hao, Z.; $\quad$ Xiu, L. Y.; Chen, S.; Ye, K.; He, S. M.; Dong, M. Q., Identification of cross-linked peptides from complex samples. Nature methods 2012, 9 (9), 904-6.

4. Lu, S.; Fan, S. B.; Yang, B.; Li, Y. X.; Meng, J. M.; Wu, L.; Li, P.; Zhang, K.; Zhang, M. J.; Fu, Y.; Luo, J.; Sun, R. X.; He, S. M.; Dong, M. Q., Mapping native disulfide bonds at a proteome scale. Nature methods 2015, 12 (4), 329-31.

5. Walzthoeni, T.; Claassen, M.; Leitner, A.; Herzog, F.; Bohn, S.; Forster, F.; Beck, M.; Aebersold, R., False discovery rate estimation for cross-linked peptides identified by mass spectrometry. Nature methods 2012, 9 (9), 901-3. 


\section{Supplementary figures}

\section{Figure S1}

a

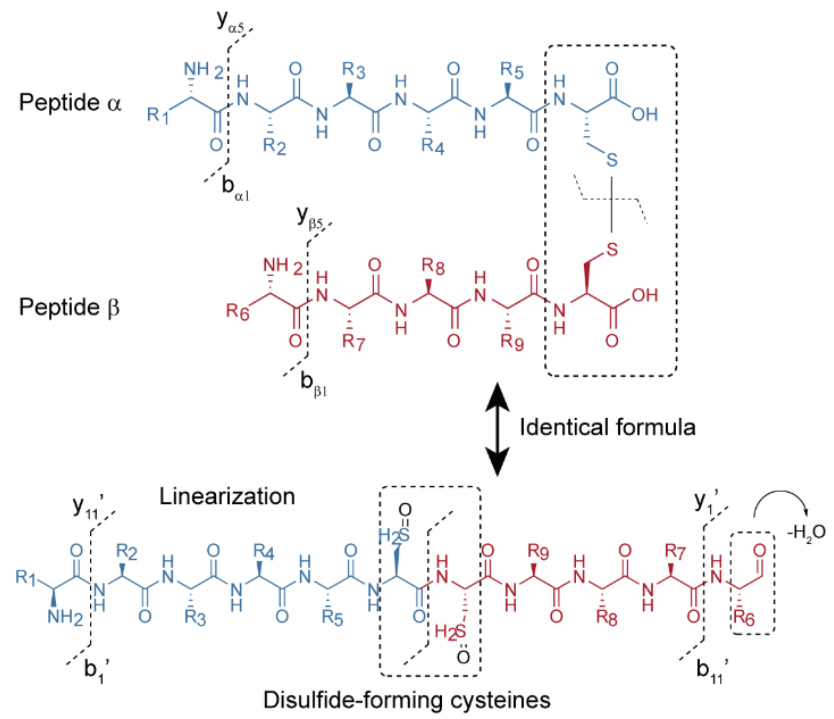

b
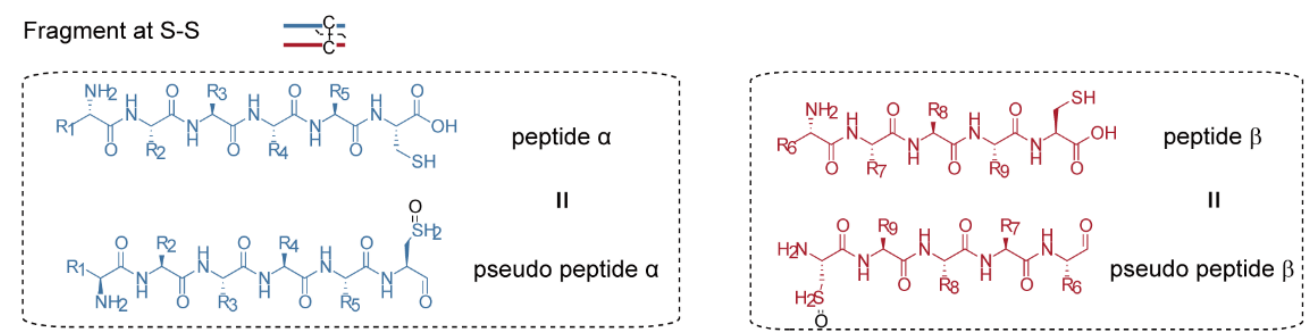

C

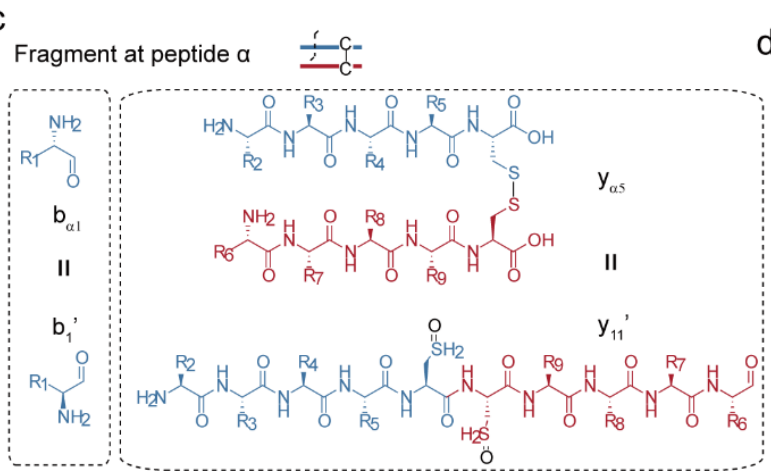

d Fragment at peptide $\beta \quad \tau_{C-}^{c-}$

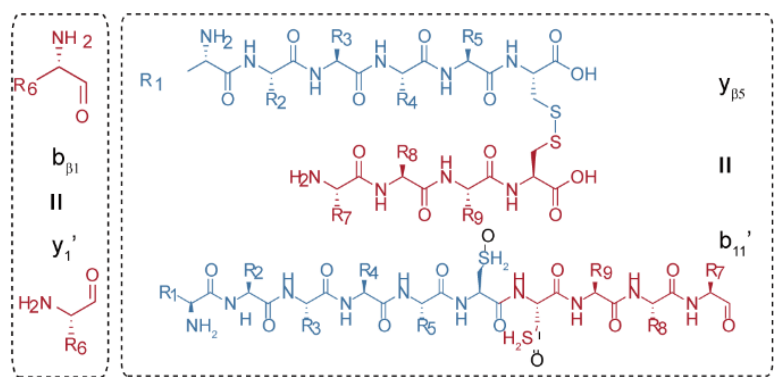

Figure S1. Linearization of disulfide-bonded peptides and their fragmentation in tandem MS.

a. Linearization of post-CPY disulfide-linked peptides. Peptide $\alpha$ keeps unchanged in the transformation, while peptide $\beta$ will become the carboxy-section of the pseudo-linear peptide. The original disulfide-linked peptides and the pseudo linearized peptide share an identical 
chemical formula. b, c and d. Fragmentation ions on pseudo linearized peptides. Ion types of b- and y-ions derived from peptides $\alpha$ keep unaffected after the linearization. The actual b- or $y$-ions will be treated as pseudo-y- or pseudo-b-ions in the linearized $\beta$ peptide, respectively. The fragmentations occurring at both peptide bond and disulfide bond are compatible with the linearization process. 


\section{Figure S2}

\begin{tabular}{cc}
\multicolumn{2}{c}{ SS bonds of P10X } \\
\hline Theoretical bonds & 55 \\
\hline CADI - Mascot & 53 \\
\hline CADI - Maxquant & 51 \\
\hline pLink 2 & 51 \\
\hline
\end{tabular}

Figure S2. Identification of disulfide bonds using different methods. The theoretical maximum number of disulfide bonds that could be formed between theses 10 peptides is 55 . The data for pLink analysis were collected from samples without CPY treatment. 


\section{Figure S3}

a

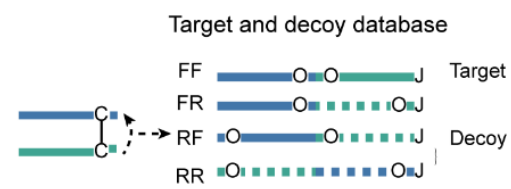

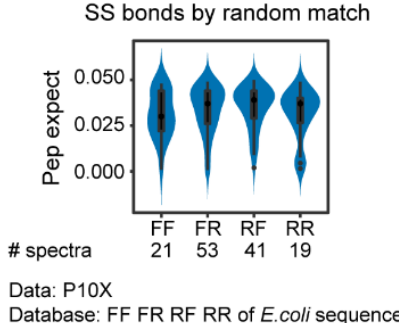

C
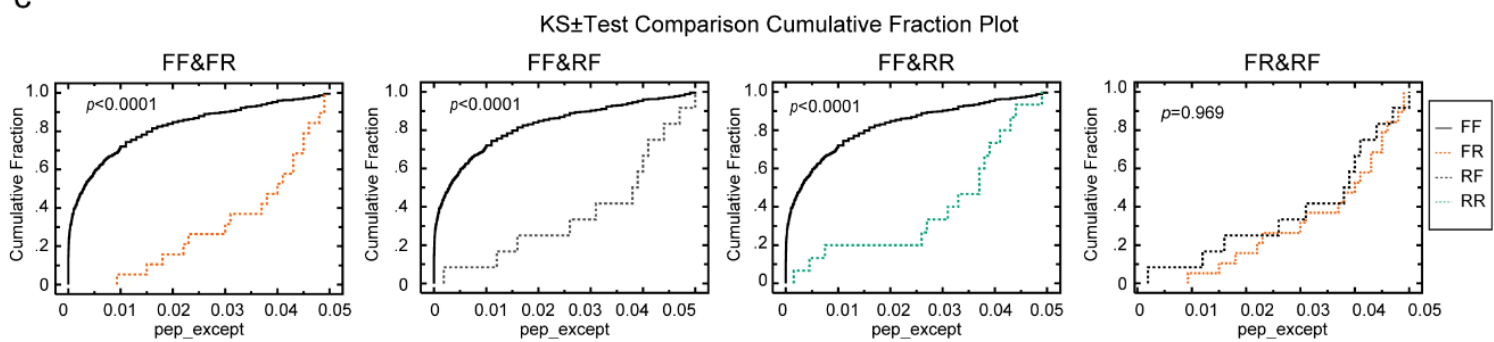

Data: P10X

Database: FF FR RF RR of E.coli sequence FF of $\mathrm{P} 10 \mathrm{X}$

d
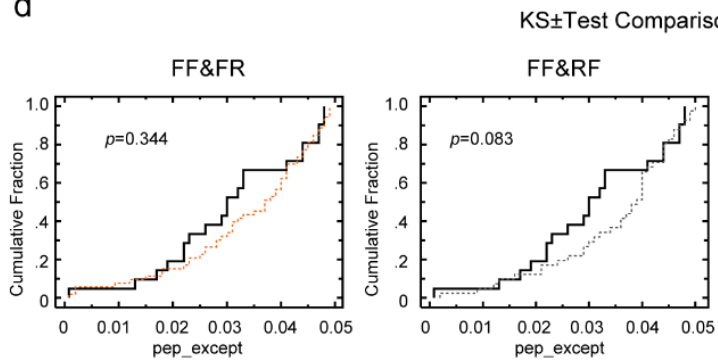

$\mathrm{KS} \pm$ Test Comparison Cumulative Fraction Plot

Data: P10X

Database: FF FR RF RR of E.coli sequence

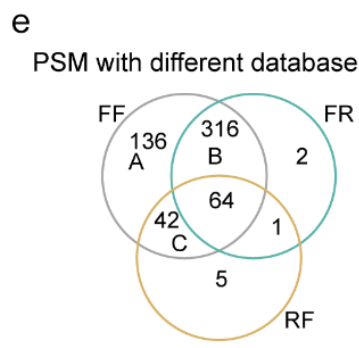

g

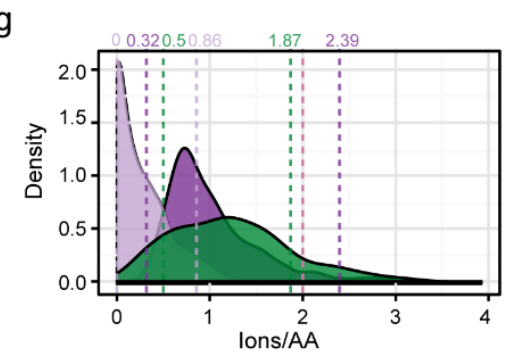

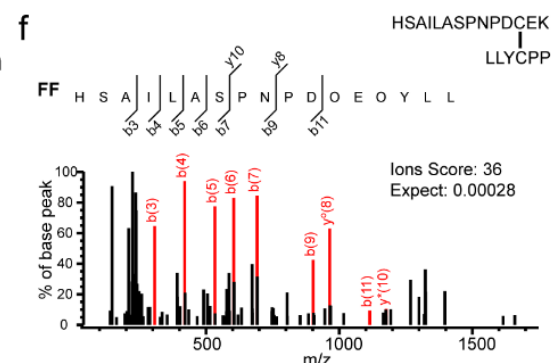

h

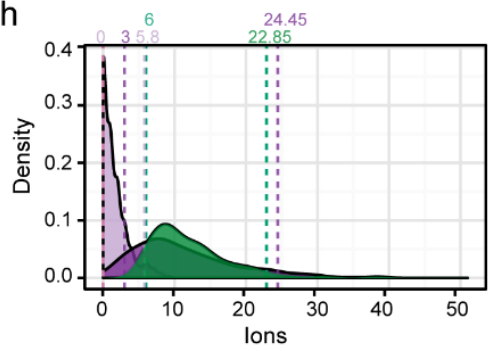

FF\&RR

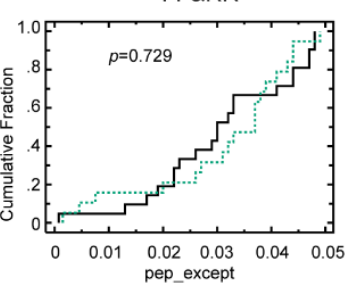

FR\&RF

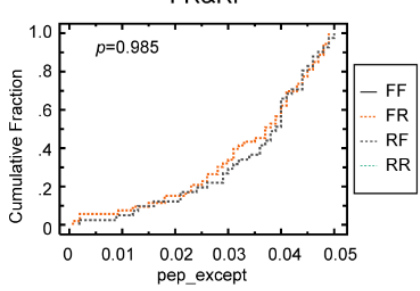


of a target database (FF). b. Random matches of P10X data in an unrelated E.coli database. The matches in all four sub-databases are random. Peptide expectation value in Mascot is used to estimate the identification confidence. c. Kolmogorov-Smirnov test for matches in different sub-databases from a search against a combination of a target database and an unrelated E.coli database. d. Kolmogorov-Smirnov test for random matches in different sub-databases from a search against an unrelated E.coli database. e. Veen diagram of matches when searching in different sub-databases separately. A same spectrum may match to FF or its hybrid forms (FR and RF) in independent searches with different sub-databases. RR returns only one hit, and is not shown in this diagram. $\mathrm{f}$ A representative MS2 shows a misidentification from a decoy database. The same spectrum is able to be identified in both FF and FR database searches. However, the reverse sequence doesn't contain any fragment ions, thus doesn't contribute to the identification. $g$ and $\mathrm{h}$. Statistics of fragment ion number and ions per amino acid of the matches in different classes. The type-A peptides have the highest confidence as they are only identified from the FF database search. The type-B and type-C peptides usually have shorter reverse parts, which contribute little to the final identifications. 


\section{Figure S4}

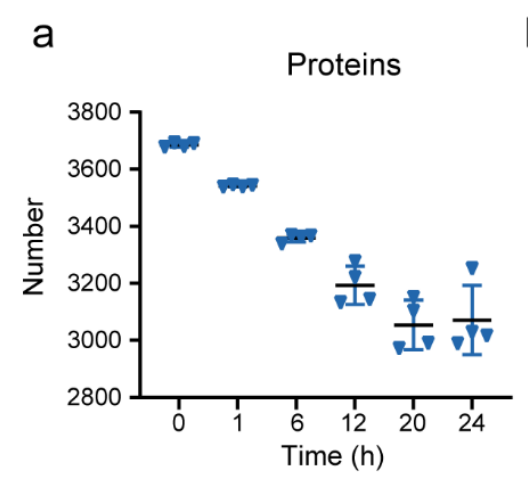

b
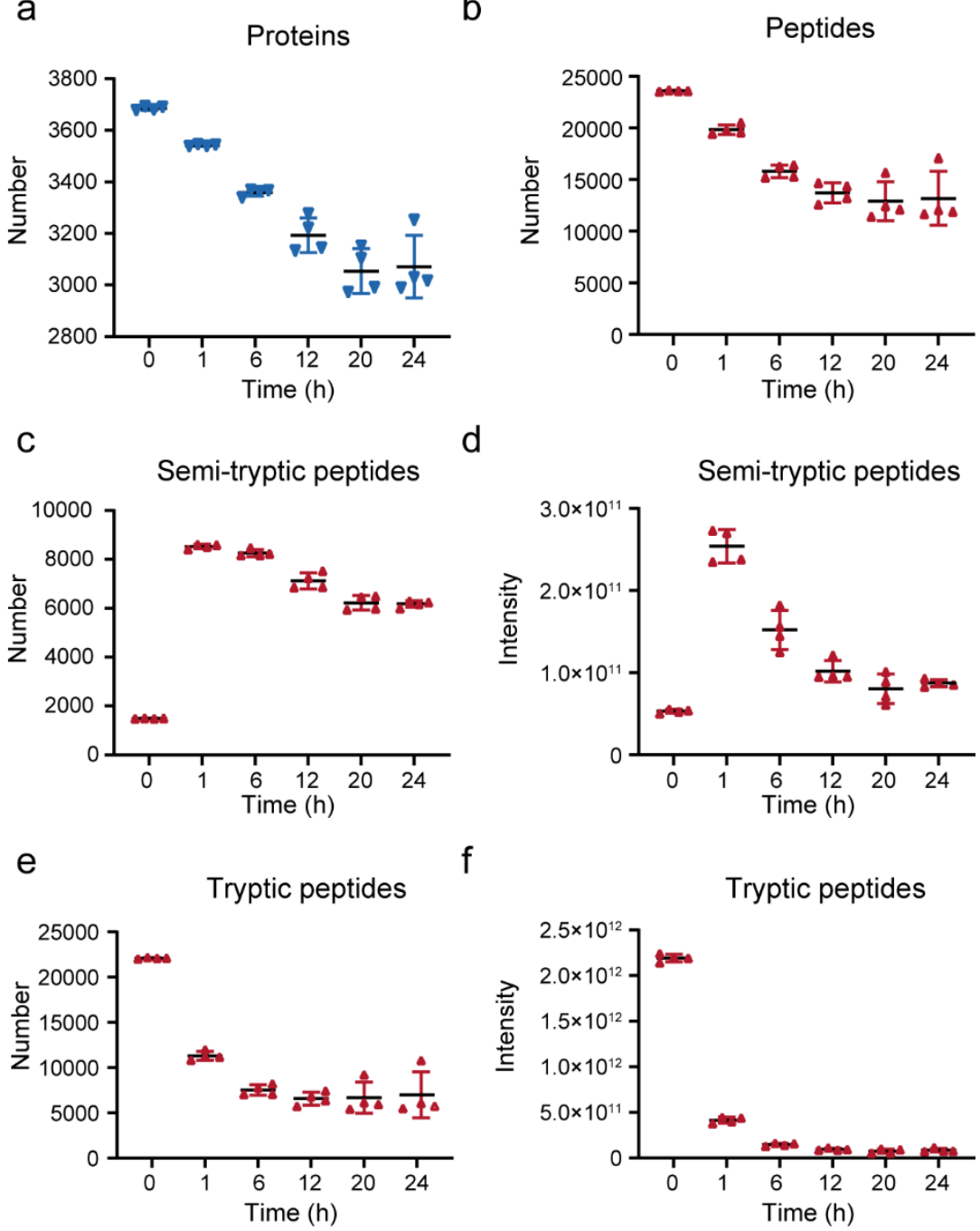

Figure S4. CADI removes linear peptides of HEK293T digest. a. Protein identification number after treating with CPY for different times. b. Peptide identification number after treating with CPY for different times. c. Semi-tryptic peptide identification number after treating with CPY for different times. The semi-tryptic peptides are mostly derived from CPY treatment. d. Summed semi-tryptic peptide intensity after treating with CPY for different times. e. Tryptic peptide identification number after treating with CPY for different times. f. Summed tryptic peptide intensity after treating with CPY for different times. 


\section{Figure S5}
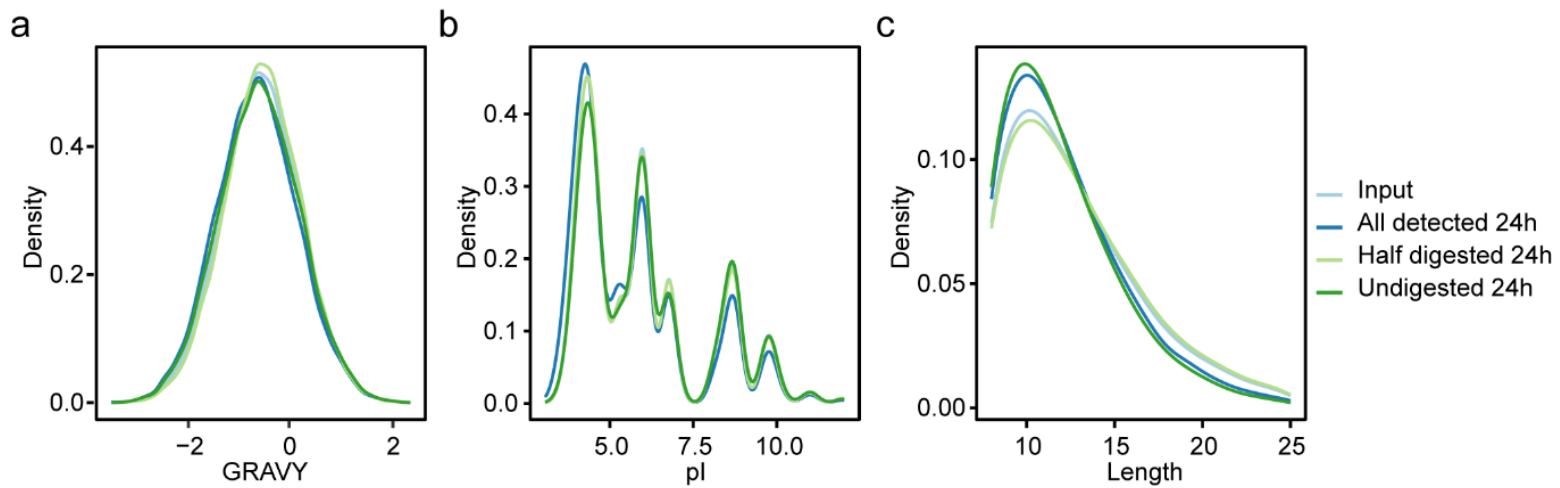

Figure S5. Comparison of peptides with different sensitivity to CPY. The four groups of peptides with different sensitivity to CPY were compared in grand average of hydropathicity index (GRAVY) (a), isoelectric point (pI) (b), and length (c). No significant difference was observed between different groups. 
Figure S6
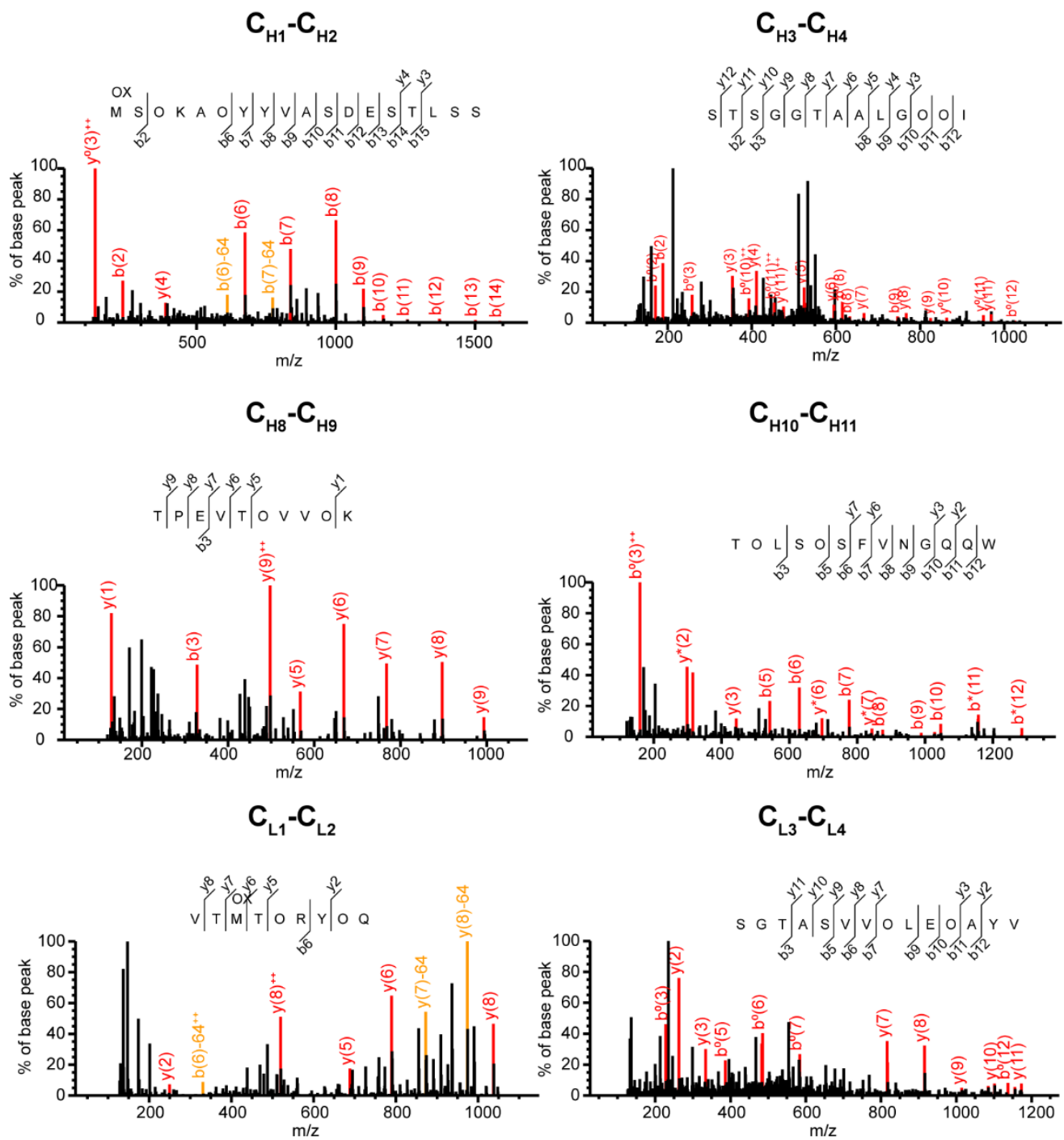

Figure S6. Tandem mass spectra of disulfide bonds from Rituximab 


\section{Figure S7}

a

RNase A C1-C7(52-121)
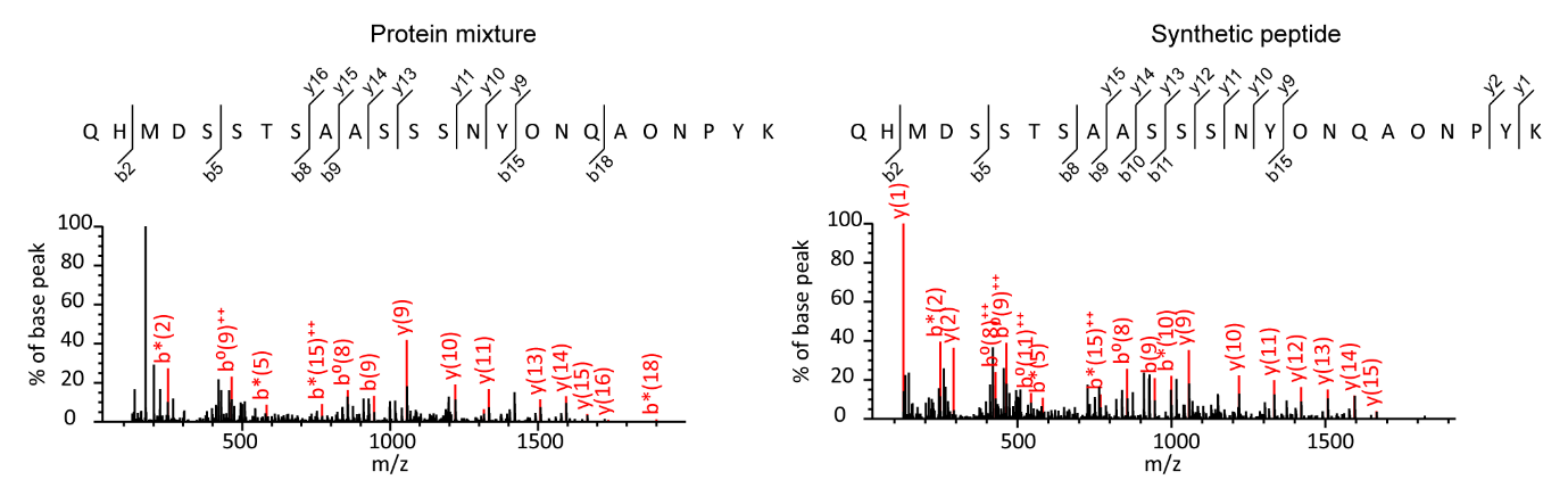

b

RNase A C1-C2(52-66)

Protein mixture
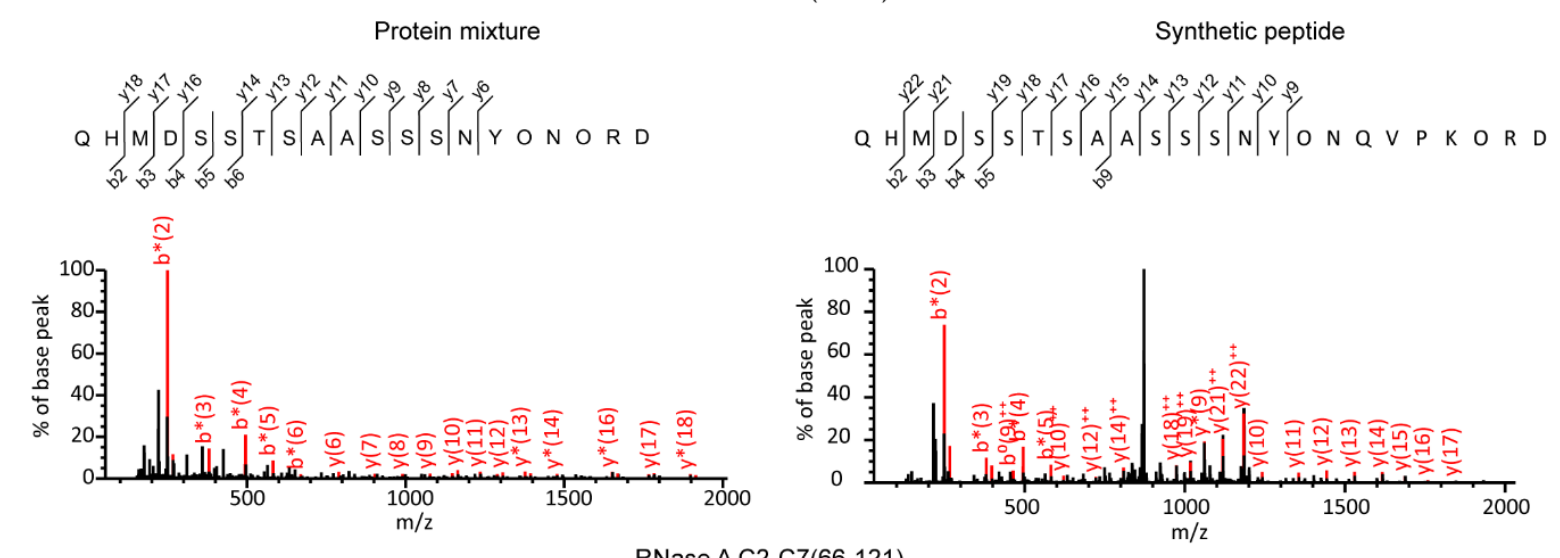

C

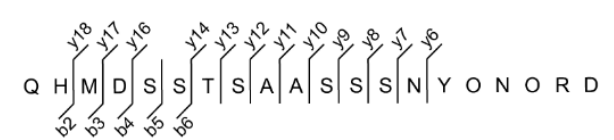

RNase A C2-C7(66-121)
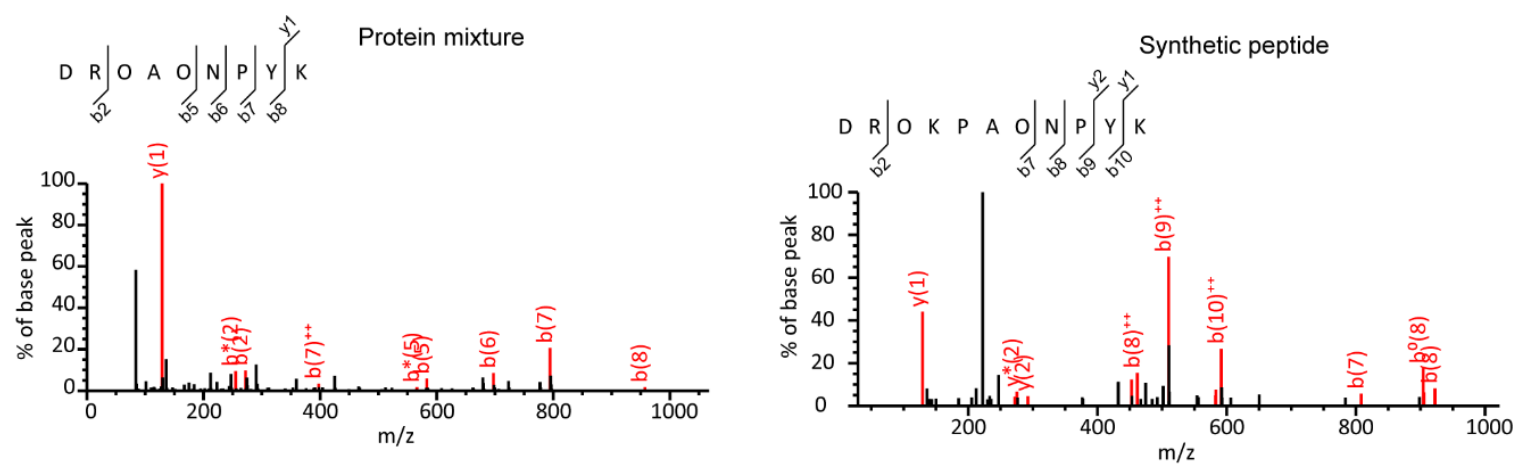

Figure S7. Validation of disulfide bonds identified from RNase A using synthetic peptides.

Three synthesized standard peptides (QHMDSSTSAASSSNYCNQMM, DRCKPVNTVHE, and KYPNCAYKTTQAN) of RNase A were disulfide linked, and analyzed by CADI. Though the left-over C-end amino acid number was changed in some synthetic peptide-formeddisulfide bonds, the fragment patterns derived from the disulfide bond-linked peptides were identical. The disulfide bonds shown in a and b were previously unknown. 


\section{Supplementary table}

Supplementary table 1: Sequences of synthetic peptides.

\begin{tabular}{cccc}
\hline NO. & Sequence & Length of peptide (aa) & Number of C-end amino acid \\
\hline 1 & TATPQQAQEVHECK & 14 & 1 \\
2 & HSAILASPNPDCEK & 14 & 2 \\
3 & TQIQSVEPCYTK & 12 & 3 \\
4 & LNEQASECEILK & 12 & 4 \\
5 & DAHNALLDICQSSGR & 15 & 5 \\
6 & ISELGAGCGGVVFK & 14 & 6 \\
7 & IDSLSACQLSQLQK & 14 & 7 \\
8 & VLTCPELYAELR & 12 & 8 \\
9 & VACVFFGGLSIK & 12 & 9 \\
10 & LLYCPPETGLFLVR & 14 & 10 \\
\hline
\end{tabular}

